\title{
Magnetization reversal mechanism of ramified and compact Co islands on $\operatorname{Pt}(111)$
}

\author{
A. Cavallin, ${ }^{*}$ F. D. Natterer, ${ }^{\dagger}$ S. Ouazi, ${ }^{*}$ G. Moulas, A. Lehnert, S. Rusponi, and H. Brune \\ Institute of Condensed Matter Physics, École Polytechnique Fédérale de Lausanne, Station 3, CH-1015 Lausanne, Switzerland
}

(Received 8 November 2013; revised manuscript received 22 September 2014; published 22 October 2014)

\begin{abstract}
We report on the magnetization reversal mechanism of Co islands on $\operatorname{Pt}(111)$ as a function of their size and shape. We measure the zero-field susceptibility $\chi(T)$ and low-temperature magnetization curves $M(H)$ with in situ magneto-optical Kerr effect. Together with the island morphology deduced from scanning tunneling microscopy, this creates sufficient information to determine both the magnetization reversal mechanism and the distribution of anisotropy energies between perimeter and surface atoms. We find a transition from quasicoherent rotation to domain wall nucleation and propagation with a critical size of 350 atoms for ramified, and of 600 atoms for compact islands.
\end{abstract}

DOI: 10.1103/PhysRevB.90.144427

PACS number(s): 75.75.Cd, 75.60.Jk, 75.75.Jn

\section{INTRODUCTION}

The understanding of thermally activated processes, in chemical reactions, diffusion, or magnetization dynamics, hangs on our knowledge of their transition state. This state is inherently short lived, making its characterization an experimental challenge. For ferromagnetic nanostructures there is at present no experimental technique combining the temporal and spatial resolution needed to capture the transition state passed upon magnetization reversal. Therefore, experimental observations are indirect, and for many magnetic nanostructures it is still an open question of how they reverse their magnetization.

The magnetic configuration of a nanostructure results from the interplay of magnetocrystalline anisotropy $K$, shape anisotropy, Heisenberg exchange energy $J$, and external parameters, such as temperature and field. Nanostructures with a ferromagnetic ground state can either reverse their magnetization by coherent rotation (CR) of all spins [1,2], by nucleation and propagation of a domain wall (DW) [3-5], or pass by other noncollinear transition states [6,7]. It is generally assumed that the transition from CR to DW takes place at a critical length $L_{\mathrm{cr}}=\pi \sqrt{A / K}$, with $A \propto J / d_{\mathrm{nn}}$ and $d_{\mathrm{nn}}$ the atomic nearest neighbor distance [8]. This expression has been derived for a cylinder and it is not clear to what extent it can be transferred to a lower dimension. If yes, one expects a shape dependence, i.e., islands with the same size reverse by CR if compact and by DW if elongated.

However, measurements unambiguously revealing the reversal mechanism are rare, and there is no clear-cut evidence of a shape dependent transition. Oblong Fe islands on Mo(110) with a size of 250 to 450 atoms have been reported to switch faster than circular ones [9]. This has been attributed to reversal by DW vs CR. However, some islands behaved as counterexamples and all had compact shapes. A much smaller threshold size of only 35 atoms for reversal by DW has been reported for Fe islands on W(110) [10]. This has

\footnotetext{
* Present address: Max Planck Institute of Microstructure Physics, Weinberg 2, 06120 Halle/Saale, Germany.

${ }^{\dagger}$ Present address: Center for Nanoscale Science and Technology, National Institute of Standards and Technology, Gaithersburg, Maryland 20889, USA.
}

been inferred from activation energies being proportional to

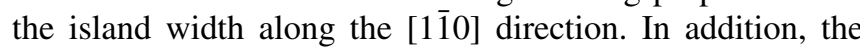
pre-exponential factors varied by orders of magnitude, which has been attributed to the varying number of DW nucleation sites. Cobalt bilayer islands on $\mathrm{Au}(111)$ are believed to reverse their magnetization by CR up to a size of 600 atoms, while a noncollinear magnon assisted process has been proposed for larger islands [7]. Finally, it was suggested that triangular Co bilayer islands on $\mathrm{Cu}(111)$ reverse by a single-element exchange spring mechanism for island sizes up to 7500 atoms, from where reversal takes place by DWs [11]. From these examples it is evident that the reversal kinetics of nanomagnets is complex and that the critical size for the reversal mechanism crossover of each transition metal element strongly depends on the substrate. Furthermore, these measurements rely on the magnetic analysis of a few dozen islands only.

In this paper we report a general method identifying the reversal mechanism for any island ensemble from temperature dependent zero-field susceptibilities $\chi(T)$ and magnetization curves $M(H)$. Both provide complementary information since the differently sized islands enter with different weight to them. Comparing measured $\chi(T)$ and $M(H)$ with numerical simulations, using the island morphologies characterized by scanning tunneling microscopy (STM) as input, enables one to determine the reversal mechanism, the critical size, and the spatial distribution of anisotropy energies within the islands. We demonstrate our method for monolayer high $\mathrm{Co}$ islands on $\mathrm{Pt}(111)$. By changing the growth conditions, we create compact islands, as well as ramified ones with long and narrow branches. The present case of Pt as substrate is particularly relevant due to the large induced spin and orbital moments and the large spin-orbit coupling resulting in very high magnetocrystalline out-of-plane anisotropy [12-15].

\section{RESULTS}

The Co islands were grown by atomic beam epitaxy on $\operatorname{Pt}(111)$ in an ultrahigh vacuum chamber combining variabletemperature STM with a highly sensitive magneto-optical Kerr effect setup [16]. The out-of-plane $\chi(T)$ was measured with an ac field of $\pm 15 \mathrm{mT}$ at $8 \mathrm{~Hz}$ [12]. Out-of-plane $M(H)$ curves were measured with a sweep rate of $9 \mathrm{mT} / \mathrm{s}$.

By controlling the growth kinetics [17] through several deposition and annealing steps at different temperatures, 
(a)
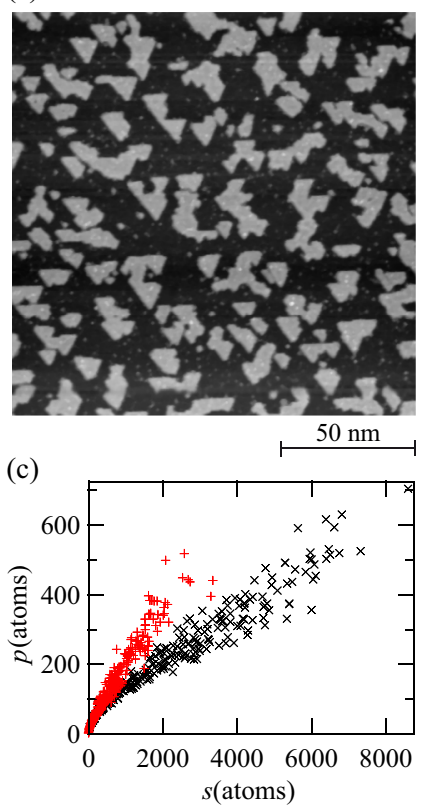

(b)

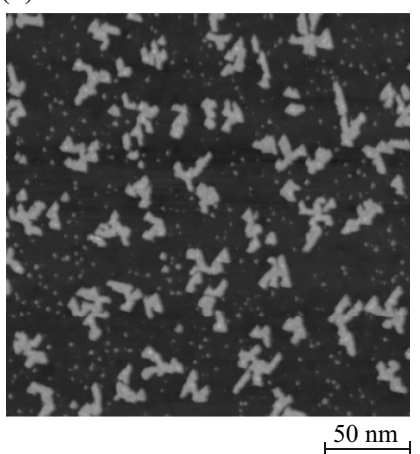

(d)

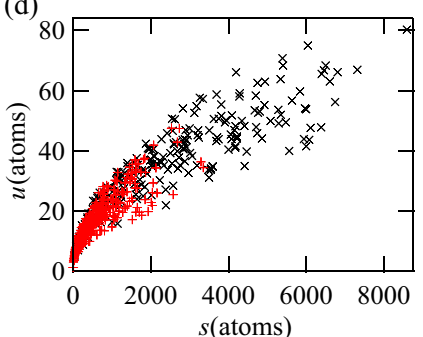

FIG. 1. (Color online) STM images of (a) compact and (b) ramified one ML high Co islands on $\mathrm{Pt}(111)$. (c) Perimeter lengths $p$ and (d) island cross sections $u$ as a function of island size $s$ for the compact (black) and ramified (red) islands for which the magnetism has been characterized in Figs. 2 and 3. [Growth parameters: (a) $\Theta=0.1 \mathrm{ML}$ at $T_{\text {dep }}=150 \mathrm{~K}, T_{\text {ann }}=270 \mathrm{~K}$ for $120 \mathrm{~s}$ followed by $\Theta=0.2 \mathrm{ML}$ at $T_{\text {dep }}=270 \mathrm{~K}$; (b) $\Theta=0.08 \mathrm{ML}$ at $T_{\text {dep }}=150 \mathrm{~K}$ followed by $\Theta=0.08 \mathrm{ML}$ at $T_{\mathrm{dep}}=250 \mathrm{~K}$; (c) compact islands as in (a) but first deposition at $T_{\text {dep }}=160 \mathrm{~K}$; and (d) ramified islands $\Theta=0.25 \mathrm{ML}$ at $T_{\text {dep }}=150 \mathrm{~K}, T_{\text {ann }}=250 \mathrm{~K}$ for $300 \mathrm{~s}$.]

we were able to create either compact or ramified islands. Figures 1(a) and 1(b) show representative STM images of both island shapes. Figures 1(c) and 1(d) show the magnetization reversal relevant morphology parameters of those islands for which the magnetic properties are characterized in Figs. 2 and 3, namely their size $s$, perimeter length $p$, and the cross section $u$ for the formation of a magnetic domain wall [18]. Each symbol is one island, and we analyzed 400 islands for each morphology class. It is evident that the amount of perimeter atoms is significantly larger for ramified than for compact islands of comparable size.

For both island shapes, and for the considered size range, the magnetic ground state is a single domain ferromagnet with an out-of-plane easy magnetization axis $[12,19,20]$. The magnetization reversal kinetics is different for the two island types, as becomes evident from comparison of Figs. 2 and 3 (black dots). Ramified islands have a much more narrow susceptibility peak that is best seen in $\chi^{\prime \prime}(T)$. They also have a significantly steeper magnetization curve enclosing a smaller area than compact ones. Numerical simulations of $\chi(T)$ and $M(H)$ with different assumptions on the reversal mechanism and anisotropy energies are shown as curves in Figs. 2 and 3 (Table I). To obtain these curves we calculate the out-of-plane component of the magnetic moment of each individual island as a function of temperature and field and sum them up to give $\chi(T)$ and $M(H)$ of the island ensemble. The ground
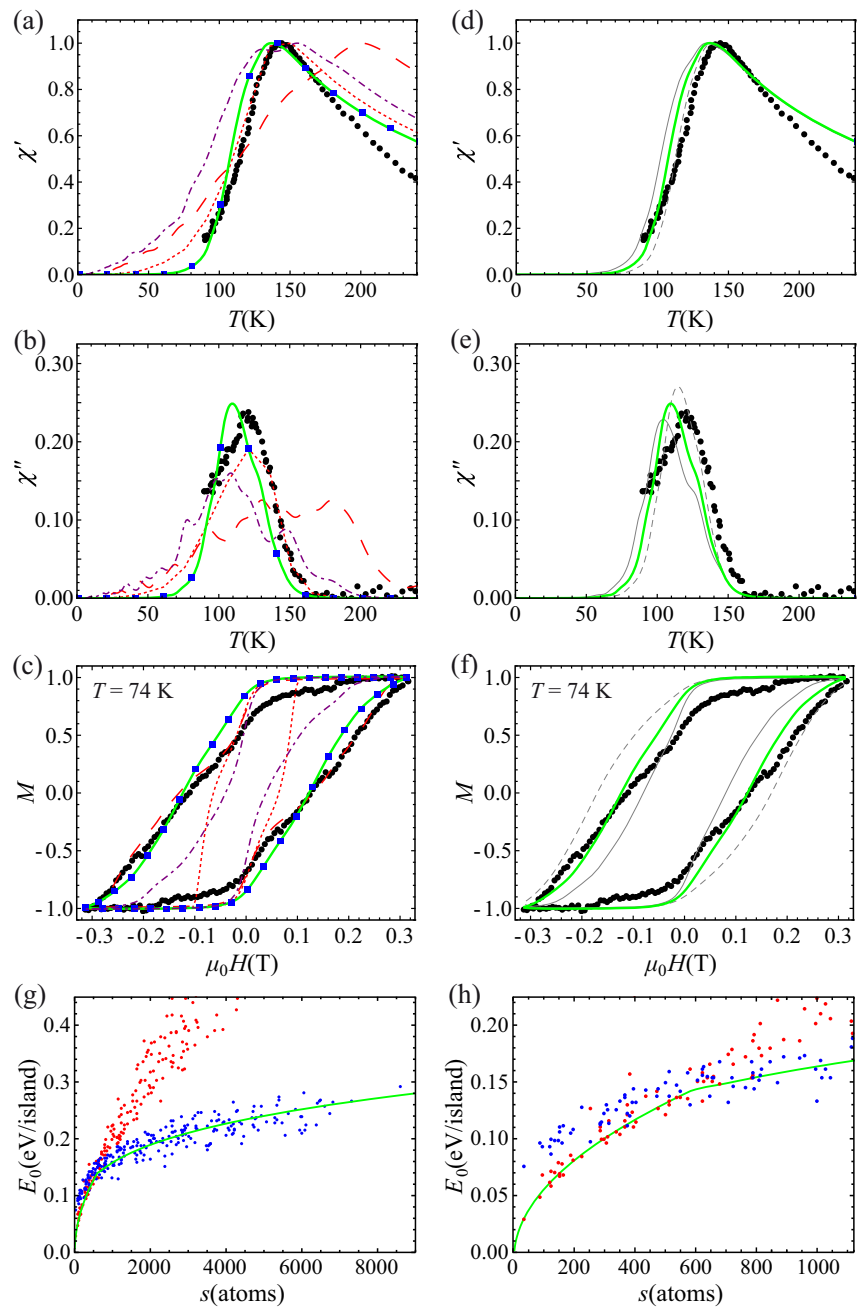

FIG. 2. (Color online) Out-of-plane zero-field susceptibility and magnetization curves of compact islands with morphology characterized by black symbols $(x)$ in Figs. 1(c) and 1(d). (a) Real and (b) imaginary part of $\chi(T)$. The peak in $\chi^{\prime}$ has been normalized to 1 thereby defining the scale for $\chi^{\prime \prime}$. (c) $M(H)$ at $T=74 \mathrm{~K}$. Measured data: black dots. (d), (e), and (f) Experimental data as in (a), (b), and (c), respectively, but different fits. Simulation curves with respective reversal mechanisms and anisotropy values are given in Table $\mathrm{I}$. $(\mathrm{g})$ Zero-field energy barriers $E_{0}$ for qCR (red) vs DW (blue) reversal. Minimizing $E_{0}$ leads to a crossover from qQR to DW at 600 atoms. (h) Zoom of the crossover region.

state out-of-plane magnetization of an island is simply given by its size $s$ times the magnetic moment per atom $m$. We take $m=2.4 \mu_{\mathrm{B}}$. This value includes the Co orbital and spin moments as well as the magnetic moment induced in the substrate $[14,15]$.

The mean out-of-plane magnetization of a given island at nonzero field and temperature is obtained by solving the master equations for the reversal kinetics. The evolution of the spin up probability $p_{\mathrm{u}}$ reads

$$
\frac{d p_{\mathrm{u}}}{d t}=p_{\mathrm{d}} v_{0} e^{-E_{\mathrm{du}} / k_{\mathrm{B}} T}-p_{\mathrm{u}} v_{0} e^{-E_{\mathrm{ud}} / k_{\mathrm{B}} T},
$$

where $E_{\mathrm{ud}}$ is the energy barrier for passing from up to down, and $E_{\mathrm{du}}$ for the other way around. We set the pre-exponential 

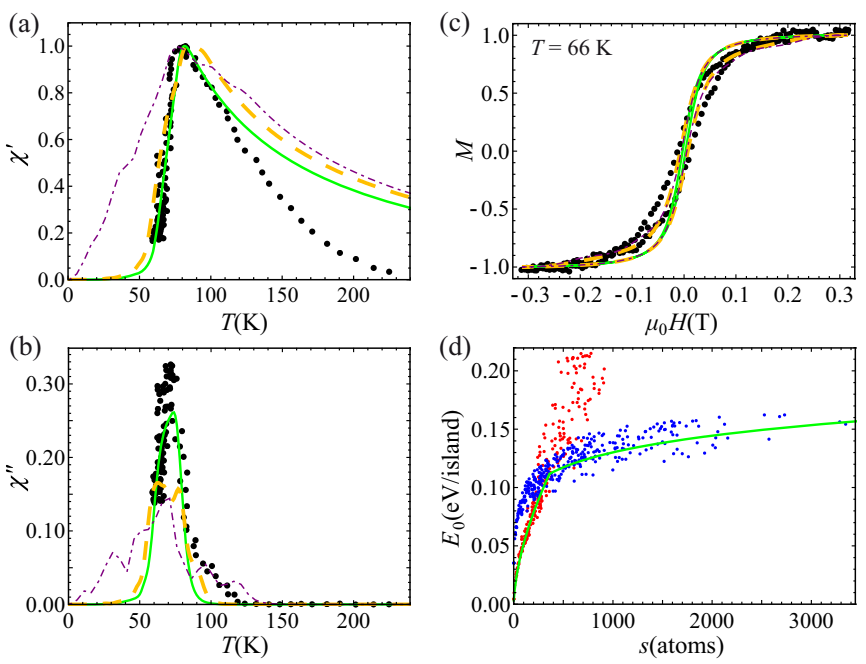

FIG. 3. (Color online) Out-of-plane zero-field susceptibility and magnetization curves of ramified islands with morphology characterized by red symbols (+) in Figs. 1(c) and 1(d). (a) Real and (b) imaginary part of $\chi$. Normalization as in Fig. 2. (c) $M(H)$ recorded at $T=66 \mathrm{~K}$. Experiments: black dots. Simulations: curves with respective reversal mechanisms and anisotropy values given in Table I. (d) Zero-field energy barriers $E_{0}$ for qCR (red) vs DW (blue) reversal. Transition from qCR to DW at 350 atoms.

factor to $v_{0}=5 \times 10^{11} \mathrm{~Hz}$ [21]. The reversal mechanism enters in the energy barriers for reversal and their field dependence. We label $E_{0}$ the zero-field value of this barrier.

For CR we have $E_{0}=s K_{\text {eff }}$ with $K_{\text {eff }}=\left[(s-p) K_{\mathrm{s}}+\right.$ $\left.p K_{\mathrm{p}}\right] / s$ accounting for the different magnetocrystalline anisotropy of surface and perimeter atoms [12]. These $K$ values include the shape anisotropy and are to a very good approximation independent of temperature in the interval of interest [22]. To describe the field dependent barriers, we introduce the reduced field $h=|H| / H_{\mathrm{c}}$, with $H_{\mathrm{c}}=2 K_{\text {eff }} / m$ [3]. For CR and the field pointing down we have $E_{\mathrm{ud}}=E_{0}(1-h)^{2}$ and $E_{\mathrm{du}}=E_{0}(1+h)^{2}$.

For the present case of islands with nonuniform $K$, the transition state during magnetization reversal at $H \neq 0$ can

TABLE I. Reversal mechanisms and anisotropy values in $\mathrm{meV}$ for the curves shown in Figs. 2 and 3. $v_{0}=5 \times 10^{11} \mathrm{~Hz}$ if not stated otherwise.

\begin{tabular}{|c|c|c|c|}
\hline In Fig. 2: & reversal model & $K_{\mathrm{s}}$ & $K_{\mathrm{p}}$ \\
\hline$-\quad-$ & $\mathrm{qCR}$ & 0.04 & 0.32 \\
\hline ........ & $\mathrm{qCR}$ & -0.03 & 1.01 \\
\hline - & DW & 0.00 & 1.50 \\
\hline & qCR-DW & 0.00 & 1.50 \\
\hline-- & $\mathrm{qCR}-\mathrm{DW}, K_{\mathrm{s}}=K_{\mathrm{p}}$ & 0.06 & 0.06 \\
\hline & qCR-DW & 0.01 & 1.15 \\
\hline--- & qCR-DW & -0.01 & 1.85 \\
\hline In Fig. 3: & reversal model & $K_{s}$ & $K_{p}$ \\
\hline & qCR-DW & -0.03 & 1.47 \\
\hline$=$ & qCR-DW, $v_{0}=5 \times 10^{13} \mathrm{~Hz}$ & 0.00 & 1.50 \\
\hline--- & $\mathrm{qCR}-\mathrm{DW}, K_{\mathrm{s}}=K_{\mathrm{p}}$ & 0.09 & 0.09 \\
\hline
\end{tabular}

be weakly noncollinear, i.e., those atoms with largest $K$ are lagging behind in the island reversal [23]. The energy barriers for this so-called quasi-CR (qCR) are obtained by considering the size and $\left(K_{\mathrm{s}}, K_{\mathrm{p}}\right)$-dependent reduction of $H_{\mathrm{c}}[23,24]$.

Figures 2(a)-2(c) reveal that one needs significantly different anisotropy parameters to fit $\chi(T)$ and $M(H)$ of compact islands with the assumption of magnetization reversal by qCR (red) for all sizes. The short-dashed curves with $K_{\mathrm{s}}=$ $-0.03 \mathrm{meV}$ and $K_{\mathrm{p}}=1.01 \mathrm{meV}$ fit $\chi^{\prime}$ and $\chi^{\prime \prime}$ very well but not at all $M(H)$, while the long-dashed curves with $K_{\mathrm{s}}=0.04 \mathrm{meV}$ and $K_{\mathrm{p}}=0.32 \mathrm{meV}$ fit $M(H)$ but not $\chi(T)$. This underlines the complementarity of the two data sets and the strength of our approach for identification of the reversal mechanism. It also shows that reversal of all island sizes by qCR can clearly be discarded. Note that the small and partly negative $K_{\mathrm{S}}$ values still imply a sizable out-of-plane (positive) magnetocrystalline anisotropy since the shape anisotropy is negative and typically $-0.12 \mathrm{meV}$ [25].

For DW reversal, $E_{0}=4 u \sqrt{A K}$, with $K=\left[(u-2) K_{\mathrm{s}}+\right.$ $\left.2 K_{\mathrm{p}}\right] / u$ the anisotropy energy density of the DW cross section $u$, again including shape and magnetocrystalline contributions. The effect of an external magnetic field pointing down is given by $E_{\text {ud }}=E_{0} f(h)$, with $f(h)=\sqrt{1-h}-h \ln (1+$ $\sqrt{1-h} / \sqrt{h})$, and $E_{\mathrm{du}}=E_{\mathrm{ud}}+2 \mu_{0} H m s[3,6,26]$.

The simulated curves for DW reversal at all island sizes are shown as blue squares and provide a good match with both $M(H)$ and $\chi(T)$ for $T<1.4 T_{\mathrm{b}}$, with $T_{\mathrm{b}}$ the blocking temperature defined as the $\chi^{\prime \prime}(T)$-peak position [the remaining discrepancies of $M(H)$ and $\chi(T)$ for higher $T$ are discussed below]. These fits yield the anisotropy values $K_{\mathrm{s}}=0.00$ and $K_{\mathrm{p}}=1.50 \mathrm{meV}$. However, if we calculate with these values $E_{0}$ for qCR (red) and DW (blue), we find that qCR has lower energy barriers up to $s_{\mathrm{cr}}=600$ atoms, from where DW takes over, see Fig. 2(h). The green curve has been calculated by taking for each island and field the mechanism with the lowest energy barrier.

Note that we have to take $A$ with $1.5 \times 10^{-11} \mathrm{~J} / \mathrm{m}$ smaller than the Co ML value of $2.3 \times 10^{-11} \mathrm{~J} / \mathrm{m}[27,28]$. This is expected for the lower dimension of the islands. It also illustrates the complementarity of $\chi(T)$ and $M(H)$ curves. While $\chi(T)$ basically determines $A K, M(H)$ does not depend on $A$ but strongly on $K$ which fixes $H_{c}$. Therefore, taking $A>1.5 \times 10^{-11} \mathrm{~J} / \mathrm{m}$ leads to smaller $K$ in order to reconcile $\chi(T)$, but this smaller $K$ yields significantly more closed $M(H)$ curves than the experiment. This shows that our data are also giving an upper limit for the effective exchange energy of the investigated system. We furthermore tested a model with uniform anisotropy. The purple dash-dotted curves clearly show that neither $\chi(T)$ nor $M(H)$ can be reproduced.

Note that the conclusions we were able to draw on the reversal mechanism, the anisotropy parameters, and the exchange energy, emerge from an all-at-once magnetic analysis of the island ensemble, in distinction with previous results that locally probed many individual magnetic islands $[10,11]$.

We now turn to the ramified islands for which $\chi(T)$ and $M(H)$ are shown in Fig. 3. For this morphology, qCR for all sizes is even less appropriate than for compact islands and therefore not shown. We show as first fit qCR-DW describing the $M(H)$ curves and $\chi(T)$ for $T<1.5 T_{\mathrm{b}}$ very well. In 
addition, these fits give with $K_{\mathrm{s}}=-0.03$ and $K_{\mathrm{p}}=1.47 \mathrm{meV}$ anisotropy values very close to the ones deduced above. This gives further confidence in the method since the mean island size and the shape are different but the system is the same and therefore large variations of the anisotropies are not expected. Looking at the $E_{0}$ values in Fig. 3(d), one infers that the critical size is now significantly smaller with $s_{\mathrm{cr}}=350$ atoms. Again, a model with uniform anisotropy shown with the purple dashed-dotted curve does not describe the data. If we constrain the anisotropy of these islands to the values of compact ones, and let the prefactor vary, we obtain the yellow dashed curve fitting $\chi(T)$ slightly worse and $M(H)$ better for $v_{0}=5 \times 10^{13} \mathrm{~Hz}$. Therefore, the conclusions drawn on the reversal mechanism and the critical size are robust against uncertainties in $v_{0}[10]$.

\section{DISCUSSION}

We first discuss two assumptions in our model, namely that islands do not interact magnetically with each other and that their Curie temperature $T_{\mathrm{C}}$ is infinite. As we will see, both are related to the remaining discrepancies between the simulated curves and our measured data.

The only sizable magnetic interactions expected between islands are dipolar ones. Their effect is different in $M(H)$ and zero-field $\chi(T)$ measurements. In the first case their strength can be estimated comparing the anisotropy field $H_{\mathrm{c}}$ of a given island with the dipolar field $H_{\mathrm{d}}$ generated by all other islands. For our system, $H_{\mathrm{c}}$ is larger than $1 \mathrm{~T}$ for all islands, while an upper limit for $H_{\mathrm{d}}$ is $40 \mathrm{mT}$, obtained in the extreme scenario of 6000 atom large islands at a distance of only $15 \mathrm{~nm}$ between their centers. Therefore, dipolar interactions are very weak. However, the observed discrepancies between the measured and modeled $M(H)$ curves can be ascribed to their characteristic antiferromagnetic character. Starting from $\left|M / M_{\text {sat }}\right|=1$ and reducing the field, the first islands that reverse their magnetization are those with small $E_{0}$. They reverse before the external field reaches zero because they are pushed by the dipolar field of the larger $E_{0}$ islands. In accordance, our measured $M(H)$ starting from saturation lies below the simulated. Also, when almost all islands have reversed, their dipolar field acts against the external field and renders the magnetic alignment of all islands more difficult. Again in accordance, we see that for the few remaining islands to switch a higher field than used in the simulated curves would be needed.

In zero-field susceptibility measurements dipolar coupling effects are strongly reduced since the sample net magnetization is nearly zero. In that case, the dipolar field manifests itself as a more shallow than $T^{-1}$ decrease of $\chi^{\prime}(T)$ above the peak temperature [29]. In marked contrast to this, we observe that for both island shapes the experimental $\chi^{\prime}(T)$ decrease above their respective peak temperatures significantly faster than $T^{-1}$ and hence they lie well below the fits.

The fact that the measured $\chi^{\prime}(T)$ of both island types lies below the simulated one for $T>1.5 T_{\mathrm{b}}$ can be ascribed to a finite $T_{\mathrm{C}}$. Figure 4 compares $\chi^{\prime}(T)$ curves for an individual island with $T_{\mathrm{C}}=\infty, 300$, and $250 \mathrm{~K}$. These values are smaller than the reported ML values of 450 and $400 \mathrm{~K}$ [14,30], which follows from the reduced exchange energy as described earlier.

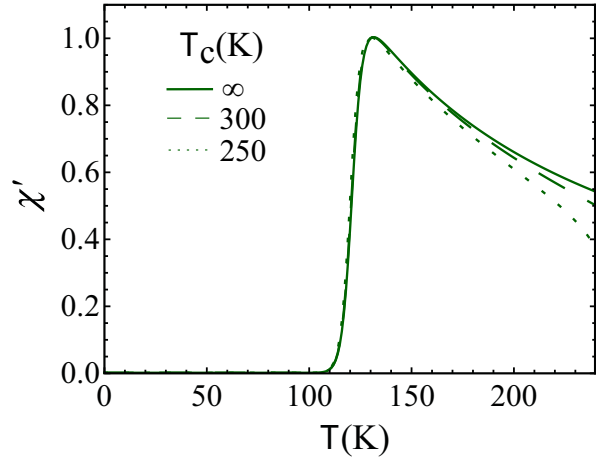

FIG. 4. (Color online) $\chi^{\prime}(T)$ curves are modified when $T$ approaches $T_{\mathrm{C}}$, as shown for a single island with $E_{0}=0.25 \mathrm{eV}$.

One sees that $\chi^{\prime}(T)$ remains unaffected up to $T=1.4 T_{\mathrm{b}}$ from where it decreases faster than the case of infinite $T_{\mathrm{C}}$. This describes our experiment very well, and it also leaves the conclusions above, that are based on the peak positions and widths of the real and imaginary part of $\chi(T)$, unaffected. In order to keep the number of parameters small, we have considered $T_{\mathrm{C}}=\infty$ in the simulations shown above. Note that a complete description would require the introduction of an island size dependent $T_{\mathrm{C}}$.

We next address the confidence intervals of $K_{\mathrm{s}}$ and $K_{\mathrm{p}}$ by looking at the energy barriers for domain wall reversal. For both compact and ramified islands, DW is the reversal process of the large island sizes contributing most to the $\chi(T)$ and $M(H)$ curves. Figure 5(a) shows the isosurfaces for $E_{0}\left(K_{\mathrm{s}}, K_{\mathrm{p}}\right)$ for an individual island with a cross section of $u=70$ atoms. The corresponding blocking temperatures can be evaluated as $T_{\mathrm{b}}=-E_{0}\left[k_{B} \ln \left(v / v_{0}\right)\right]^{-1}$, see Fig. 5(b), thus tracing $\chi^{\prime}$ and $\chi^{\prime \prime}$ peak temperature shifts. Clearly $K_{\mathrm{s}}$ and $K_{\mathrm{p}}$ are strongly anticorrelated, and very small variations of the first entail large variations of the second to maintain the $\chi$ peaks at the same temperature. Moving along iso- $T_{b}$ lines, the peak width in $\chi(T)$ and the $M(H)$ width are seen to vary, see gray curves in Figs. 2(d)-2(f). A precise evaluation of the quality of the agreement between experimental data and simulated curves is given by the sum of the squared residuals of $\chi^{\prime}(T), \chi^{\prime \prime}(T)$, and $M(H)$. We obtain $9.7,4.1$, and 9.8 for $\left(K_{\mathrm{s}}, K_{\mathrm{p}}\right)$ equal to $(-0.01,1.85),(0.00,1.50)$, and $(0.01,1.15)$, respectively. In passing, we note that imposing uniform anisotropy $K_{\mathrm{s}}=K_{\mathrm{p}}$ returns a value of 24.7 in the best case.

The above analysis is based on the island morphologies characterized by means of STM. Evidently this sample is smaller than the one characterized by MOKE. Increasing the ensemble size of the STM measurements would result for each of the two island morphologies in a single smooth curve for the island size distribution $N_{\mathrm{s}}$. However, the distributions of $p$ and $u-N_{\mathrm{p}}$ and $N_{\mathrm{u}}$, respectively - that enter the magnetic simulations have an intrinsic width since Figs. 1(c) and 1(d) show that islands of the same size have different perimeter lengths and domain wall widths. We can assess the effect of including a larger statistical ensemble into the magnetic simulations by approximating the size distributions as a Gaussian, see the full curve in Fig. 6(a) in comparison with the dots showing the experiment (Table II). For the magnetic simulations it is important to get the large island sizes right 

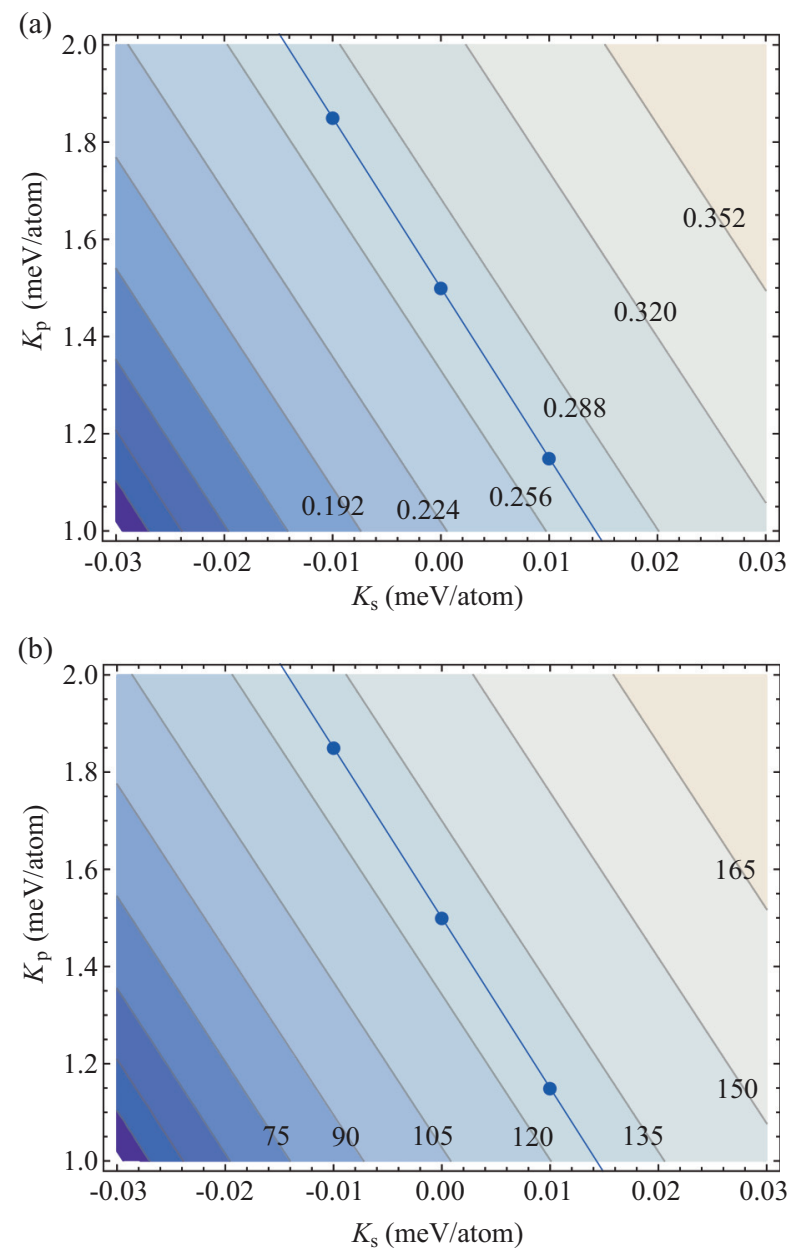

FIG. 5. (Color online) (a) DW $E_{0}\left(K_{\mathrm{s}}, K_{\mathrm{p}}\right)(\mathrm{eV})$ for a single island with $u=70$ atoms, and (b) corresponding $T_{b}\left(K_{\mathrm{s}}, K_{\mathrm{p}}\right)(\mathrm{K})$. The blue dots indicate the qCR-DW model parameters returning the green and gray curves in Fig. $2,\left(K_{\mathrm{s}}, K_{\mathrm{p}}\right)=(-0.01,1.85),(0.00,1.50)$, and $(0.01,1.15) \mathrm{meV} /$ atom.

since they dominate the signal, and therefore the deviations between Gaussian and experiment at small sizes are irrelevant. If we then include $N_{\mathrm{p}}(s)$ and $N_{\mathrm{u}}(s)$ with widths matching the ones found in the ensemble probed by STM, we reproduce to within $<2 \%$ our simulations that were based on islands for which the individual $s, p$, and $u$ values were mapped out. These curves are shown as green curves and are almost identical with the ones shown in Fig. 2. Deviations become significant, however, if we neglect the intrinsic widths of $N_{\mathrm{p}}$ and $N_{\mathrm{u}}$, and assume instead single $p$ and $u$ values for islands with the same size. The pink long-dashed curves in Fig. 6, obtained with all other simulation parameters identical to the ones derived above, agree slightly less well with experiment. More importantly, neglecting the $N_{\mathrm{p}}$ and $N_{\mathrm{u}}$ widths narrows the $E_{0}$ distribution and thus $\chi(T)$. The distribution of energy barriers at $0.25 \mathrm{~T}, E_{0.25 \mathrm{~T}}$, is similarly affected with the consequence of a steeper $M(H)$ reversal at high fields, see Fig. 6(c). We note that technically one can compensate these trends to some extent by introducing new $K_{\mathrm{s}}$ and $K_{\mathrm{p}}$ values, see the orange short-dashed curves. However, the physics of our system incorporates the distributions $N_{\mathrm{p}}$ and $N_{\mathrm{u}}$ with finite
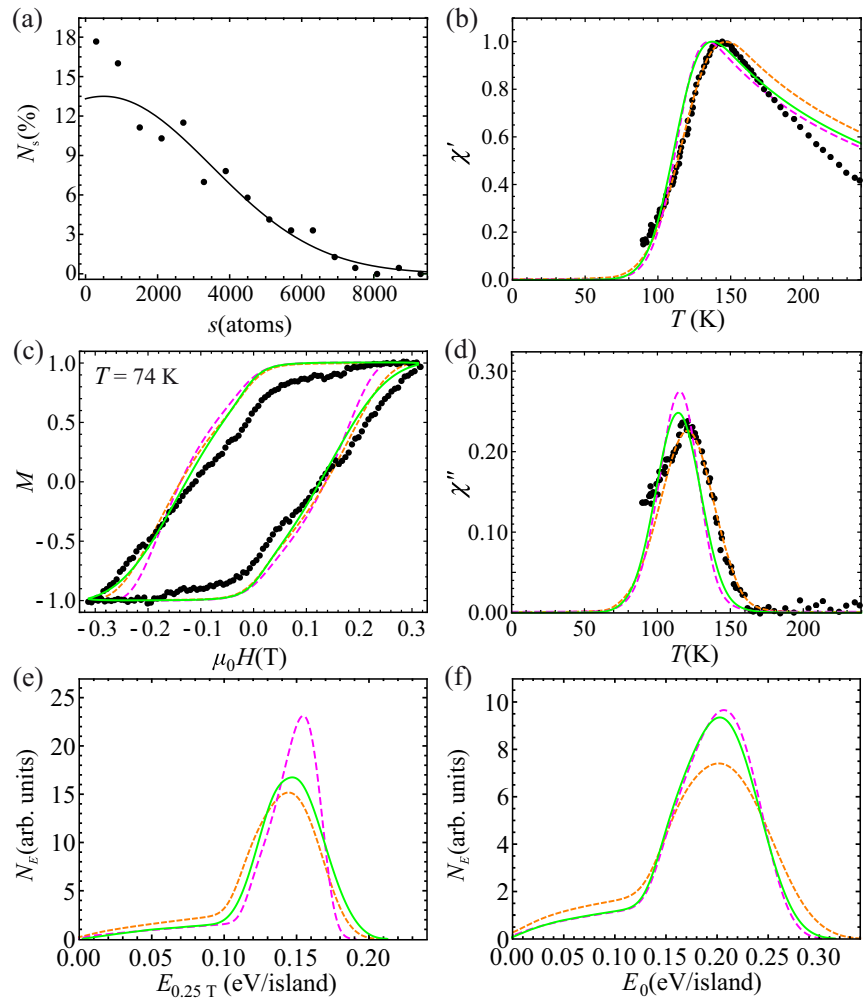

FIG. 6. (Color online) Compact islands: (a) measured size distribution and relative Gaussian model, $N(s) \propto e^{-\left(\frac{s-500}{3000}\right)^{2}}$. (b), (c), and (d) Experimental data and green curve as in Figs. 2(a), 2(b), and 2(c), respectively, with additional magnetic simulations. Simulation curves with respective morphology $s, p, u$ input data and anisotropy values are given in Table II. (f) and (e) $E_{0}$ and $E_{0.25} \mathrm{~T}$ distributions, respectively.

widths and therefore the green activation energy distributions for magnetization reversal are the most realistic ones.

Concerning the shape dependence of the critical size, it is important to realize that $E_{\mathrm{DW}}(u(s))$ show similar trends for the two morphologies, since the underlying $u(s)$ do, see Fig. 1(d). Therefore, any difference must depend on $E_{\mathrm{CR}}(s, p(s))$. At any given size, $E_{\mathrm{CR}}(s, p(s))$ is larger for ramified islands because they have more atoms at the perimeter that are found to have more anisotropy. This increases the barriers for CR of ramified islands with respect to those of compact islands, while the barriers for DW are largely shape independent with the result that the crossover to DW happens for ramified islands at smaller sizes.

TABLE II. Morphology $s, p, u$ input data, anisotropy values in meV for the curves shown in Fig. 6. $v_{0}=5 \times 10^{11} \mathrm{~Hz}$.

\begin{tabular}{|c|c|c|c|}
\hline In Fig. 6: & qCR-DW reversal model & $K_{\mathrm{s}}$ & $K_{\mathrm{p}}$ \\
\hline & Gaussian $N_{\mathrm{s}}$, see Fig. 6(a) & 0.00 & 1.50 \\
\hline & $N_{\mathrm{p}}(s), N_{\mathrm{u}}(s)$, see text & & \\
\hline & $\begin{array}{l}\text { Gaussian } N_{\mathrm{s}} \text {, see Fig. 6(a) } \\
p(s)=1.63 s^{0.65}, u(s)=1.01 s^{0.47}\end{array}$ & 0.00 & 1.50 \\
\hline -ש-n & $\begin{array}{l}\text { Gaussian } N_{\mathrm{s}} \text {, see Fig. 6(a) } \\
p(s)=1.63 s^{0.65}, u(s)=1.01 s^{0.47}\end{array}$ & 0.024 & 1.05 \\
\hline
\end{tabular}


Finally, we test the general expressions for the critical length of the crossover from CR to DW for islands with compact morphology. The expression in the Introduction, using a mean value of $K=0.15 \pm 0.04 \mathrm{meV}$ and $A$ as above, yields $L_{\mathrm{cr}}=9.4 \pm 1.3 \mathrm{~nm}$. For islands with $s_{\mathrm{cr}}=600$ atoms we estimate from our STM images a size of $L_{\mathrm{cr}}=9 \pm 2 \mathrm{~nm}$ in excellent agreement with the general expression. A somewhat smaller value of $L_{\mathrm{cr}}=w \pi / 2=6.3 \mathrm{~nm}$ is deduced from the static domain wall width $w$ of room temperature grown Co ribbons attached to $\mathrm{Pt}(111)$ steps [20].

\section{CONCLUSIONS}

We introduced a method to reliably deduce from the temperature dependence of the zero-field susceptibility and from magnetization curves of island ensembles, both taken along the easy magnetization direction, the anisotropy energies of surface and perimeter atoms, the reversal mechanisms, the critical sizes for the transition in reversal mechanism, and the dependence of this size on island shape. We demonstrated for
$\mathrm{Co} / \mathrm{Pt}(111)$ the growth of ramified and compact morphologies for comparable island sizes. We identified a transition from quasicoherent rotation to domain wall nucleation and propagation with shape dependent critical size. As expected, ramified islands start to reverse by domain wall nucleation and propagation at smaller sizes than compact ones. Our method can be applied to any system for which STM morphology data are combined with in situ measurements of $\chi(T)$ and $M(H)$. The shape dependence of the critical size is expected to be stronger in systems with a more homogeneous anisotropy distribution.

\section{ACKNOWLEDGMENTS}

We gratefully acknowledge fruitful discussions with H. B. Braun and V. Repain and collaboration in the early stages of this work with S. Rohart. We further acknowledge funding from the Swiss National Science Foundation and from the Sino-Swiss Science and Technology Cooperation Project No. IZLCZ2 123892.
[1] W. F. Brown, Phys. Rev. 130, 1677 (1963).

[2] W. Wernsdorfer, E. B. Orozco, K. Hasselbach, A. Benoit, B. Barbara, N. Demoncy, A. Loiseau, H. Pascard, and D. Mailly, Phys. Rev. Lett. 78, 1791 (1997).

[3] H. B. Braun, Phys. Rev. Lett. 71, 3557 (1993).

[4] W. Wernsdorfer, B. Doudin, D. Mailly, K. Hasselbach, A. Benoit, J. Meier, J. P. Ansermet, and B. Barbara, Phys. Rev. Lett. 77, 1873 (1996).

[5] H. B. Braun, J. Appl. Phys. 99, 08F908 (2006).

[6] H. B. Braun, J. Appl. Phys. 85, 6172 (1999).

[7] S. Rohart, P. Campiglio, V. Repain, Y. Nahas, C. Chacon, Y. Girard, J. Lagoute, A. Thiaville, and S. Rousset, Phys. Rev. Lett. 104, 137202 (2010).

[8] Here $K$ is per unit cell volume, otherwise we give $K$ per atom.

[9] M. Bode, O. Pietzsch, A. Kubetzka, and R. Wiesendanger, Phys. Rev. Lett. 92, 067201 (2004).

[10] S. Krause, G. Herzog, T. Stapelfeldt, L. Berbil-Bautista, M. Bode, E. Y. Vedmedenko, and R. Wiesendanger, Phys. Rev. Lett. 103, 127202 (2009).

[11] S. Ouazi, S. Wedekind, G. Rodary, H. Oka, D. Sander, and J. Kirschner, Phys. Rev. Lett. 108, 107206 (2012).

[12] S. Rusponi, T. Cren, N. Weiss, M. Epple, P. Buluschek, L. Claude, and H. Brune, Nat. Mater. 2, 546 (2003).

[13] P. Gambardella, S. Rusponi, M. Veronese, S. S. Dhesi, C. Grazioli, A. Dallmeyer, I. Cabria, R. Zeller, P. H. Dederichs, K. Kern, C. Carbone, and H. Brune, Science 300, 1130 (2003).

[14] G. Moulas, A. Lehnert, S. Rusponi, J. Zabloudil, C. Etz, S. Ouazi, M. Etzkorn, P. Bencok, P. Gambardella, P. Weinberger, and H. Brune, Phys. Rev. B 78, 214424 (2008).

[15] A. Lehnert, S. Dennler, P. Bloński, S. Rusponi, M. Etzkorn, G. Moulas, P. Bencok, P. Gambardella, H. Brune, and J. Hafner, Phys. Rev. B 82, 094409 (2010).

[16] A. Lehnert, P. Buluschek, N. Weiss, J. Giesecke, T. Treier, S. Rusponi, and H. Brune, Rev. Sci. Instrum. 80, 023902 (2009).
[17] H. Brune, Surf. Sci. Rep. 31, 125 (1998).

[18] $u$ is defined as the smallest cross section of a given island since the activation energy of domain wall nucleation and propagation is proportional to $u$, hence the smallest cross section of a given island defines its magnetization reversal rate.

[19] T. Cren, S. Rusponi, N. Weiss, M. Epple, and H. Brune, J. Phys. Chem. B 108, 14685 (2004).

[20] F. Meier, K. von Bergmann, P. Ferriani, J. Wiebe, M. Bode, K. Hashimoto, S. Heinze, and R. Wiesendanger, Phys. Rev. B 74, 195411 (2006).

[21] We calculated $M(H)$ curves with the expected field dependence of $v_{0}$, however, the differences are barely visible such that we restrict the discussion to a constant $v_{0}$ for simplicity.

[22] For a uniaxial system $K(T)=K(0)[M(T) / M(0)]^{3}$, see Ref. [31], and $M(T)=[(w(T)+1) /(w(T)-1)]^{3 / 8}[(w(T)-$ $3) /(w(T)+3)]^{1 / 8}$, where $w(T)=\exp \left[\ln (3) T_{\mathrm{c}} / T\right]$ for an Ising model on a triangular lattice, see Ref. [32], an adequate approximation for a bistable system on a fcc (111) surface. Assuming $T_{\mathrm{c}}=300 \mathrm{~K}$, see Ref. [14], this translates in a maximum variation of $K(T)$ of $2 \%$ between $T=170$ and $0 \mathrm{~K}$, and of $5 \%$ between $T=200$ and $0 \mathrm{~K}$.

[23] S. Rohart, V. Repain, A. Thiaville, and S. Rousset, Phys. Rev. B 76, 104401 (2007).

[24] Energy barrier calculations for spin-wave assisted reversal, see Ref. [7], are computationally very demanding. They are feasible for a few hundred atom large islands. However calculations of spin-wave assisted reversal on the vast range of sizes and morphologies investigated in this study are impossible with present computer resources.

[25] S. Ouazi, S. Vlaic, S. Rusponi, G. Moulas, P. Buluschek, K. Halleux, S. Bornemann, S. Mankovsky, J. Minár, J. B. Staunton, H. Ebert, and H. Brune, Nat. Commun. 3, 1313 (2012).

[26] H. B. Braun and N. H. Bertram, J. Appl. Phys. 75, 4609 (1994). 
[27] S. Bornemann, O. Šipr, S. Mankovsky, S. Polesya, J. B. Staunton, W. Wurth, H. Ebert, and J. Minár, Phys. Rev. B 86, 104436 (2012).

[28] The Co ML on $\operatorname{Pt}(111)$ value is $J=18 \mathrm{meV}$, see Ref. [27], identical with $A=2.3 \times 10^{-11} \mathrm{~J} / \mathrm{m}$.

[29] R. W. Chantrell, N. Walmsley, J. Gore, and M. Maylin, Phys. Rev. B 63, 024410 (2000).
[30] O. Šipr, S. Bornemann, J. Minár, S. Polesya, V. Popescu, A. Šimůnek, and H. Ebert, J. Phys.: Condens. Matter 19, 096203 (2007).

[31] H. B. Callen and E. Callen, J. Phys. Chem. Solids. 27, 1271 (1966).

[32] D. A. Lavis and G. M. Bell, Statistical Mechanics of Lattice Systems (Springer, Berlin, 1999), Vol. 1. 